University of Nebraska - Lincoln

DigitalCommons@University of Nebraska - Lincoln

Food and Drug Administration Papers

U.S. Department of Health and Human Services

2020

\title{
Long-term negative emotional outcomes of warzone TBI
}

Jennifer J. Vasterling

VA Boston Healthcare System, Boston \& Boston University School of Medicine, Boston \& VA Boston

Healthcare System, jennifer.vasterling@va.gov

Mihaela Aslan

VA Cooperative Studies Program \& Yale University, New Haven

Susan P. Proctor

U.S. Army Research Institute of Environmental Medicine, Natick \& VA Boston Healthcare System, Boston

John Ko

VA Cooperative Studies Program

Xenia Leviyah

VA Boston Healthcare System

See next page for additional authors

Follow this and additional works at: https://digitalcommons.unl.edu/usfda

Part of the Dietetics and Clinical Nutrition Commons, Health and Medical Administration Commons, Health Services Administration Commons, Pharmaceutical Preparations Commons, and the Pharmacy Administration, Policy and Regulation Commons

Vasterling, Jennifer J.; Aslan, Mihaela; Proctor, Susan P.; Ko, John; Leviyah, Xenia; and Concato, John, "Long-term negative emotional outcomes of warzone TBI" (2020). Food and Drug Administration Papers. 52.

https://digitalcommons.unl.edu/usfda/52

This Article is brought to you for free and open access by the U.S. Department of Health and Human Services at DigitalCommons@University of Nebraska - Lincoln. It has been accepted for inclusion in Food and Drug Administration Papers by an authorized administrator of DigitalCommons@University of Nebraska - Lincoln. 


\section{Authors}

Jennifer J. Vasterling, Mihaela Aslan, Susan P. Proctor, John Ko, Xenia Leviyah, and John Concato 


\title{
Long-term negative emotional outcomes of warzone TBI
}

\author{
Jennifer J. Vasterling ${ }^{a, b, c}$, Mihaela Aslan ${ }^{d, e}$, Susan P. Proctor ${ }^{f, g}$, John Ko ${ }^{d}$, Xenia \\ Leviyah $^{c}$ and John Concato ${ }^{\mathrm{e}, \mathrm{h}}$ \\ ${ }^{\mathrm{a} P s y c h o l o g y}$ Service, VA Boston Healthcare System, Boston, MA, USA; ${ }^{\mathrm{b}}$ Department of Psychiatry, \\ Boston University School of Medicine, Boston, MA, USA; ${ }^{C}$ National Center for PTSD, VA Boston \\ Healthcare System, Boston, MA, USA; ${ }^{\mathrm{d} C l i n i c a l}$ Epidemiology Research Center, VA Cooperative \\ Studies Program, West Haven, CT, USA; ${ }^{e}$ Department of Medicine, School of Medicine, Yale

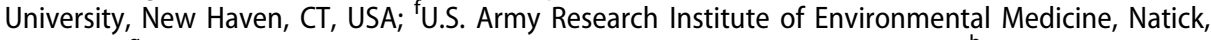

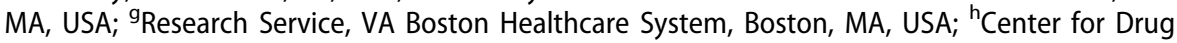 \\ Evaluation and Research, Food and Drug Administration, Silver Spring, MD, USA
}

\begin{abstract}
Objective: Many veterans of the Iraq and Afghanistan Wars have experienced traumatic brain injury (TBI). Although prior work has examined associations between TBI and development of psychiatric syndromes, less is known about associations between TBI and component emotions constituting these syndromes, especially in the long term. The purpose of this study was to examine the long-term emotional consequences of deployment-related TBI.

Methods: As part of VA Cooperative Studies Program \#566, we assessed a sample of $n=456$ US Army soldiers prior to an index deployment to Iraq, and again an average of 8.3 years $(S D=2.4$ years) after their deployment for a long-term follow-up assessment. In this report, we used adjusted regression analyses to examine the relationship of deployment TBI to depression, anxiety, and stress symptom severity measured at the long-term follow-up assessment. A structured interview was used to determine TBI history; the Depression, Anxiety, and Stress Scale, 21-item version (DASS-21) was used to determine emotional status at the follow-up evaluation.

Results: Warzone TBI events, particularly when greater than mild in severity, were independently associated with depression, anxiety, and stress severity at long-term follow-up, even after taking into account variance attributable to pre-deployment emotional distress and war-zone stress. Post-hoc analyses did not detect independent associations of either number of events or injury mechanism with outcomes.

Conclusions: These findings highlight the potentially enduring and multi-faceted emotional effects of deployment TBI, underscoring the need for early assessment of negative affectivity in warzone veterans reporting TBI.
\end{abstract}

\section{ARTICLE HISTORY}

Received 24 July 2019

Accepted 5 March 2020

Published online 17 April

2020

\section{KEYWORDS}

Depression; anxiety; stress; military; head injury

CONTACT Jennifer J. Vasterling jennifer.vasterling@va.gov @ Psychology Service, VA Boston Healthcare System, Boston, MA, USA

This work was authored as part of the Contributor's official duties as an Employee of the United States Government and is therefore a work of the United States Government. In accordance with 17 U.S.C. 105, no copyright protection is available for such works under U.S. Law. 
Military personnel deployed to the Iraq and Afghanistan war zone are at increased risk of traumatic brain injury (TBI). From 2000 through the first quarter of 2018, over 380,000 US service members were diagnosed with TBI (Defense and Veterans Brain Injury Center, retrieved June 2019, https://dvbic.dcoe.mil/dod-worldwide-numbers-tbi). Many, but not all, of these injuries were incurred during operational deployments, and most $(82.3 \%)$ were classified as mild in severity.

TBI in both military and civilian contexts, even at milder levels of severity, may result in at least short-term emotional distress or dysregulation (e.g. Ma et al., 2019; Shields et al., 2016). Depression and anxiety commonly follow TBI (Smith, 2006; van der Horn et al., 2013), and several prospective studies have shown that mild TBI increases risk of posttraumatic stress disorder (PTSD)-a disorder constituted by both anxiety and dysphoria-within the year following TBI (Bryant et al., 2010; Vasterling et al., 2018), and also over multi-year periods (O'Donnell et al., 2016; Stein et al., 2019; Vasterling et al., 2018). Emotional distress may impede recovery from TBI (Lingsma et al., 2015; Ponsford et al., 2012; van der Naalt et al., 2017; Wäljas et al., 2015) and has been associated with less adaptive coping strategies (Shields et al., 2016), cognitive (Stulemeijer et al., 2007) and other post-concussive symptoms (Waldron-Perrine et al., 2014), poorer vocational outcomes (Church et al., 2019; van der Horn et al., 2013), more difficulty with community and psychosocial re-integration (Draper et al., 2007; Williams et al., 2014), and reduced quality of life (Schiehser et al., 2015).

Much of the military TBI literature addressing emotional correlates of TBI has focused on diagnostic syndromes such as PTSD (e.g. Vasterling et al., 2018). Less attention has been devoted to understanding associations between military TBI and disturbances of the component emotions of psychiatric disorders, and especially whether emotional distress persists over time. Examination of component emotions both helps to clarify brain-behavior relationships between TBI and basic emotional states and permits further elucidation of the key emotional mechanisms associated with development and maintenance of clinical psychiatric syndromes.

In this study, we focused on negative affectivity. Reflecting Clark and Watson (1991)'s tripartite conceptualization of negative affect, we examined anxiety, depression, and stress as long-term outcomes of deployment-related TBI in a study population of current and former US Army Soldiers who were followed since prior to an index Iraq War deployment that was the first operational deployment for the majority of the sample. The availability of data relevant to emotional distress that was collected prior to the $\mathrm{TBI}$ event provides a rare opportunity to examine long-term emotional outcomes of warzone TBI adjusted for pre-deployment distress.

More specifically, drawing on data from Department of Veterans Affairs (VA) Cooperative Study \#566 (CSP\#566), in a large sample constituted in 2003 as part of the Neurocognition Deployment Health Study (NDHS; Vasterling et al., 2006), we examined depression, anxiety, and stress as dimensional constructs of negative affectivity an average of over 8 years subsequent to an index deployment to Iraq. We hypothesized that deployment TBI, adjusted for demographic factors, deployment stress exposures, and pre-deployment emotional distress, would be associated with higher levels of depression, anxiety, and stress, as compared with no TBI. Because greater disruption of neural structures and networks associated with emotion 
regulation (e.g. Newsome et al., 2013; Spikman, Timmerman, Milders, Veenstra, \& van der Naalt, 2012) and greater symptom burden (e.g. Swan et al., 2018) would be expected as TBI severity increases, we hypothesized that participants who reported that their most severe TBI event was at least of moderate severity would report greater distress compared with participants who reported their most severe TBI event to be of mild severity.

\section{Materials and methods}

\section{Sampling and recruitment}

Human subjects approval was obtained from the VA Central Institutional Review Board. Documentation of written consent was waived; all participants provided phone consent.

The analytic sample for the current study draws on data provided by CSP\#566, representing a component of a longitudinal cohort study (NDHS; Vasterling et al., 2006) that includes pre-deployment, post-deployment, and long-term follow-up (CSP\#566) assessments conducted in reference to an index Iraq War deployment for each participant. To achieve occupational and geographic dispersion within the warzone, NDHS participants were sampled at the battalion level, as US Army regular active duty and activated reservist soldiers prior to their deployment to Iraq (see Vasterling et al., 2016 for details).

As a nationally dispersed cohort comprised of service members and military veterans at the time of long-term follow-up, CSP\#566 participants were recruited individually from the $n=1,120$ surviving NDHS cohort members who (a) provided consent during previous NDHS assessments to be contacted for future research, (b) deployed $\geq 30$ days in support of Operation Iraqi Freedom at least once subsequent to their baseline NDHS assessment, (c) provided valid self-report, indicated by the absence of invalid response patterns (i.e. uniform endorsement of unidirectional extreme responses on psychometric measures in which the pathologic response varied in direction), and (d) had accessible Defense Manpower Data Center military records (see Aslan et al., 2013 for more details). Only participants yielding valid response profiles from the long-term follow-up were included in the final CSP\#566 analytic sample $(n=598)$.

Participants from the CSP\#566 analytic sample were excluded from analyses in the current study if they: (a) reported warzone TBI history inconsistently (i.e. changed their response from endorsement of TBI to non-endorsement, or vice-versa) across the postdeployment and long-term follow-up assessments $(n=52)$, or (b) did not complete the measures relevant to key constructs, including emotional distress, TBI, and stress exposure $(n=45)$. Finally, because this paper focuses specifically on warzone TBI, we further excluded participants who reported civilian or military TBI events outside the context of warzone deployment $(n=45)$, yielding an analytic sample of $n=456$.

\section{Data sources}

Pre-deployment and post-deployment assessments, occurring an average of 117.8 days ( $S D=96.6$ days) prior to deployment and 110.4 days ( $S D=80.6$ days) after return from deployment, respectively, were conducted in-person at military installations. Primary 
long-term follow-up data used in the current analyses were gathered by phone interview and mail surveys, occurring an average of 8.3 years $(S D=2.4$ years), after return from the index deployment. Deployment information was verified by Defense Manpower Data Center records.

\section{Negative affectivity}

Negative emotional states were measured with the Depression, Anxiety, and Stress Scale, 21-item version (DASS-21; Lovibond \& Lovibond, 1995). The DASS-21 is based on a tripartite model of emotion that posits that depression and anxiety can be differentiated by specific components (high physiological arousal for anxiety and low positive affectivity for depression) (Clark \& Watson, 1991). The DASS-21 yields three subscales measuring the dimensions of depression (e.g. "I felt down-hearted and blue"), anxiety (e.g. "I experienced trembling"), and stress (e.g. "I found it difficult to relax") and has been shown to have strong psychometric properties for each of the subscales, including good convergent, discriminant, and construct validity, as well as high internal consistency (Henry \& Crawford, 2005). Construct validity has likewise been supported in TBI samples (Randall et al., 2017). Internal consistency in our sample was high for each scale (Cronbach's $\alpha=0.93,0.84$, and 0.91, for depression, anxiety, and stress, respectively).

\section{Pre-deployment emotional distress}

To account for the emotional status of participants prior to deployment, we created a composite score from the following pre-deployment measures, all administered via written survey at the pre-index deployment assessment: PTSD Checklist, civilian version (PCL-C) summary score (Ruggiero et al., 2003); Mental Component Summary (MCS) score of the Veterans 12-Item Health Survey, or VR-12 (Kazis et al., 1999; Selim et al., 2009); and Profile of Mood States (POMS) subscale summary scores (McNair et al., 1971) for tension-anxiety, depression, anger-hostility, vigor-activity, fatigue, and confusion-bewilderment.

\section{Deployment stress exposures}

Because combat and other war-zone exposures (e.g. participation in convoys) may increase risk of deployment-related TBI, we ascertained Iraq War deployment stress exposures via written survey. Specifically, combat exposure and exposure to war-zone events other than direct combat during deployment were measured with summary scores using the Combat Experiences and Post-Battle Experiences modules, respectively, from the Deployment Risk and Resilience Inventory (DRRl; King et al., 2006; Vogt et al., 2008). Given that these two scales were highly correlated in our sample $(r=0.76)$, and to reduce the number of predictors considered in the multivariate model, the two scales were combined-as they were in previous studies of this cohort (Ciarleglio et al., 2018; Vasterling et al., 2015). Each scale consists of 16 dichotomous items (experienced/non-experienced), which were added to give a single cumulative index of combat and post-battle experiences (range $=0$-32). Higher scores indicated greater exposure to war-zone combat and war-zone events; internal consistency was high for the combined scale (Cronbach's $\alpha=0.92$ ). Most participants $(n=322$ and $n=323$, 
respectively, for the Combat Experiences and Post-Battle Experiences scales) provided report of their war-zone stress exposures at the post-deployment assessment. Deployment stress exposures were assessed at long-term follow-up ( $n=131$ and $n=129$, respectively, for the Combat Experience and Post-Battle Experiences scales) for those participants who completed their index deployment subsequent to the NDHS post-deployment assessment or who did not complete the warzone stress exposure scales during the post-deployment assessment.

\section{TBI history}

Because TBI events in the war-zone were not systematically captured within military records at the time of the index deployments (2003-2005), TBI history was ascertained by structured interview. As described elsewhere (Alosco et al., 2016), the long-term follow-up interview, conducted by phone by a doctoral-level clinical psychologist, documented lifetime history of TBI events associated with either a) alteration of consciousness (explained as "dazed" or loss of memory for "what was happening during, immediately before, or immediately after the injury"), or b) loss of consciousness. Characteristics of the five most serious injuries for each participant were documented, including TBI experienced during the index deployment. TBI classification required head injury or blast exposure ("injuries to your head or close exposures to explosive blasts"), with alteration or loss of consciousness. Each TBI event was categorized as mild or greater than mild, based on commonly used categorizations for duration of loss of consciousness (mild: < 30 minutes) and posttraumatic amnesia (mild: < 24 hours). Mild, complicated TBI (i.e. TBI events with $<30$ minutes loss of consciousness and $<24$ hours posttraumatic amnesia, but with skull fracture or requiring surgery, were grouped with greater than mild TBI events). To establish reliable reporting of $\mathrm{TBI}$ events occurring during the index deployment, we examined TBI report obtained at long-term follow-up in the context of archived interview data obtained from a post-deployment TBI interview that queried for TBI events associated with loss of consciousness. Specifically, and as described under "Sampling and Recruitment," we included only those participants who reported TBI events with loss of consciousness reliably across assessments.

\section{Statistical analyses}

Data were analyzed using SAS v9.4 (SAS Institute Inc, Cary, NC, USA) and Spotfire $\mathrm{S}+$ v8.2 (TIBCO Software Inc, Palo Alto, CA, USA). Item-level missing data were not imputed for primary outcomes (i.e. DASS-21 scores). For other variables, item-level missing data were imputed only if $<50 \%$ of items were missing on a given scale. Values for missing DRRI Combat Experiences and Post-battle Experiences scales, involving $<5 \%$ and $<2 \%$ of participants, respectively, and for each POMS subscale, involving $<2 \%$ of participants across scales, were imputed case-wise using the mean value of the remaining completed items on that scale. Values for each missing PCL-C item, involving $<4 \%$ of participants, were also imputed using the mean value of each PCLC DSM-IV-TR symptom cluster (Criteria B, C, D) for that person. No cases were missing $>50 \%$ of the items relevant to each PTSD symptom cluster. Values for missing VR-12 
MCS subscale were imputed according to a regression algorithm published by the scale developers (Selim et al., 2009).

Principal components analysis was used to derive a variable describing emotional health status at the pre-deployment assessment for use as an adjustment factor in the regression models. The PCL-C, CES-D, VR-12 MCS, and six POMS subscale scores were combined into a single principal component that accounted for most of the variance in the original variables. A factor score was constructed using the first principal component, which was the only component with an Eigenvalue $>1$ (Eigenvalue $=5.72$ ). VR-12 MCS and POMS vigor subscale scores were reversed, so that higher scores on all variables indicate presence of more symptomology.

To examine associations between index deployment TBI (none, mild, greater than mild) and emotion outcomes at long-term follow-up, evaluated separate hierarchical linear regression models for each DASS-21 variable (depression, anxiety, stress). To narrow potential adjustment factors that we selected based on prior literature to those associated with emotional distress as measured by DASS-21 scores, we examined bivariate correlations between candidate covariates (age, self-reported ethnicity, education, and military duty status at long-term follow-up; military rank, military occupation type, and emotional distress at pre-deployment assessment; deployment stress exposure) and DASS-21 scores. The first step of the model included the factors that were found to be significantly associated with outcomes in the bivariate correlations: education and duty status (veteran, reservist, regular active duty) at long-term followup, pre-deployment emotional distress, deployment stress exposure, and time interval from end of deployment to long-term follow-up. The second step of the model examined the independent contributions of deployment TBI (none, mild, greater than mild), following adjustment for the variables included in the first step. To control for multiple comparisons across three outcomes, we applied a Bonferroni correction (0.05/3), to yield a significance level of $p<.017$.

\section{Results}

\section{Sample characteristics}

Sample characteristics are presented in Table 1. Of the $n=456$ participants, $62.5 \%$ ( $n=285)$ reported no history of TBI, whereas $23.5 \%(n=107)$ reported at least one mild TBI during the index deployment, and $14.0 \%(n=64)$ reported at least one TBI of greater than mild severity during the index deployment. In the overall sample, the majority of participants were men, Caucasian, military veterans, and had completed at least part college at long-term follow-up. Participants with history of greater than mild $\mathrm{TBI}$ reported more emotional distress at the pre-deployment assessment than those with history of mild TBI or no TBI. Participants with combat arms occupational specialties at pre-deployment were more likely than those with combat support or service support specialties to incur a TBI during deployment. Relatedly, incurring a greater than mild TBI was associated with the most extensive war-zone stress exposure, with mild TBI associated with an intermediate level of war-zone stress exposure, and no TBI associated with the least war-zone stress exposure. A similar pattern emerged on DASS-21 measures at long-term follow-up-participants with greater than mild TBI 


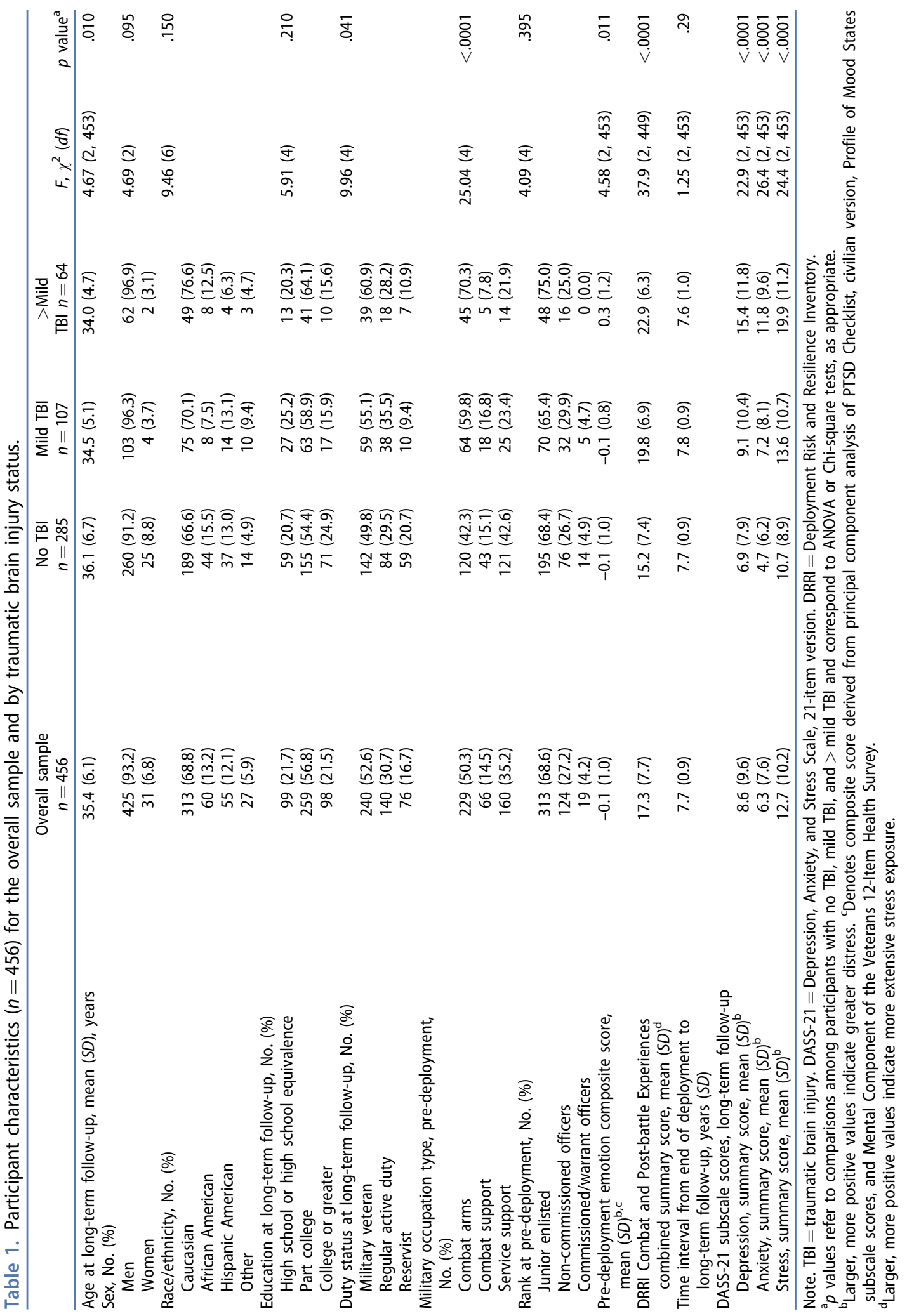


reported the greatest emotional distress, those with mild TBI reporting intermediate levels of distress, and those with no TBI history reporting the least distress. At longterm follow-up, participants with $\mathrm{TBI}$ history were also younger, and more likely to have separated from military service, than those without TBI history.

\section{TBI characteristics}

Details regarding TBI history are presented in Table 2. Of the 171 participants reporting a deployment TBI, the majority (62.6\%) reported their "most serious" event to be mild. Most participants reported experiencing two or fewer total TBI events over their lifetimes. Among participants whose most serious TBI was mild, 55.1\% reported experiencing only one event; among those whose most serious TBI was greater than mild, $32.8 \%$ reported experiencing one event. All of the most recent events were post-acute ( $>3$ months) at the time of long-term follow-up assessment.

\section{Associations of TBI with emotion outcomes}

The first step of each of the linear regression models examining associations of adjustment factors with DASS-21 measures were significant $\left(R^{2}=0.164, p<.0001\right.$ for depression; $R^{2}=0.127, p<.0001$ for anxiety; $R^{2}=0.187, p<.0001$ for stress). No indication of multicollinearity (variance inflation factors: 1.04-1.18) was found among variables entered in the first step of the models. The addition of TBI in the second step explained an increase of $33.5 \%$ in the variance for depression beyond the percent variance explained by the first step: new $R^{2}=0.219 ; \Delta R^{2}=0.219$ minus $0.164=0.055$ $(p<.0001)$. Similar calculations when adding TBI to the models indicated an increase

Table 2. Characteristics by severity level of the most significant deployment-related traumatic brain injury $^{\mathrm{a}}(n=171)$.

\begin{tabular}{|c|c|c|}
\hline & $\begin{array}{c}\text { Mild } n=107 \\
\quad \text { No. (\%) }\end{array}$ & $\begin{array}{c}>\text { Mild } n=64 \\
\quad \text { No. }(\%)\end{array}$ \\
\hline \multicolumn{3}{|l|}{ Deployment for which participant reported TBI } \\
\hline Index deployment only & $66(61.7)$ & $30(46.9)$ \\
\hline Subsequent deployment only & $22(20.6)$ & $11(17.2)$ \\
\hline Both index and subsequent deployment & $19(17.7)$ & $23(35.9)$ \\
\hline \multicolumn{3}{|l|}{ Number of TBI events reported across all deployments } \\
\hline 1 & $59(55.1)$ & $21(32.8)$ \\
\hline 2 & $24(22.4)$ & $10(15.6)$ \\
\hline 3 & $8(7.5)$ & $15(23.5)$ \\
\hline 4 & $6(5.6)$ & $8(12.5)$ \\
\hline 5 or more & $10(9.4)$ & $10(15.6)$ \\
\hline \multicolumn{3}{|l|}{ TBI mechanism } \\
\hline Blunt force only/blunt force with blast $(n=101)$ & $55(51.4)$ & $46(71.9)$ \\
\hline Blast only $(n=70)$ & $52(48.6)$ & $18(28.1)$ \\
\hline \multicolumn{3}{|c|}{ Time, most recent TBI event to long-term follow-up assessment } \\
\hline \multicolumn{3}{|l|}{$<1$ month } \\
\hline \multicolumn{3}{|l|}{$1-3$ months } \\
\hline$>3$ months -1 year & $3(2.8)$ & \\
\hline$>1$ year -2 years & $1(0.9)$ & \\
\hline$>2$ years & $103(96.3)$ & $64(100)$ \\
\hline
\end{tabular}

$\mathrm{TBI}=$ traumatic brain injury.

aparticipants reporting civilian TBI were excluded from the sample. 
of $46.5 \%$ in the variance for anxiety $\left(\Delta R^{2}=0.059, p<.0001\right)$, and an increase of $20.5 \%$ in the variance for stress $\left(\Delta R^{2}=0.038, p<.0001\right)$.

As shown in Table 3 for the fully-adjusted model, deployment TBI was significantly associated with DASS-21 depression, anxiety, and stress severity at long-term followup. Specifically, in contrast to no TBI, the association of mild TBI with DASS-21 anxiety met the Bonferroni-corrected level of significance, whereas associations of mild TBI with DASS-21 depression and stress approached but did not reach the corrected significance level. Greater than mild TBI was significantly associated with each of the DASS-21 measures (depression, anxiety, and stress). In addition, it is noteworthy that TBI was significantly associated with greater emotional distress at long-term follow-up, even after adjustment for pre-deployment distress, which was highly correlated with each emotional outcome in both steps of the model (all $p<.0001$ ). Also, in the both steps of the model, military veteran status, as compared with active duty status, was significantly associated with greater depression ( $p<.0001$ in both steps), anxiety ( $p=.003$ in both steps), and stress ( $p=.006$ in Step $1 ; p=.007$ in Step 2), at long-term follow-up.

We conducted a sensitivity analysis, including only those participants who provided self-report of deployment stressors at the post-deployment assessment $(n=324)$, to assure that potential recall bias among participants providing retrospective reports of relatively remote deployment stressors did not unduly influence results. The pattern of results of this analysis was the same as that for the full sample. In the final step of the model, TBI remained significantly associated with depression (mild TBI: $p=.021$; greater than mild TBI: $p<.0001$ ), anxiety (mild TBI: $p=.005$ ); greater than mild TBI: $p$ $<.0001$ ), and stress (mild TBI: $p=.017$; greater than mild TBI: $p=.0004$ ), with relatively stronger effects for greater than mild TBI as compared with mild TBI.

\section{Post-hoc analyses within the TBI subsample $(n=171)$}

To further explore the relationship of TBI characteristics with emotions as long-term follow-up, we conducted two sets of exploratory post-hoc analyses, examining the number of $\mathrm{TBI}$ events and the mechanism of injury in relation to outcomes.

\section{Associations of number of TBI events with emotional outcomes}

Partial correlations adjusted for covariates, to capture greater neural burden as measured by the number of TBI events experienced by each participant, revealed that number of TBI events was not significantly associated with emotional distress at long-term follow-up (depression: $r=0.10, p=.22$; anxiety: $r=0.05, p=.55$; stress: $r=0.10, p=.22$ ).

\section{Associations of TBI mechanism with emotional outcomes}

As shown in Table $2,59.1 \%$ of the sample $(n=101)$ reported that their most significant TBI involved blunt force only $(n=23)$ or blunt force plus blast $(n=78)$, whereas $40.9 \%(n=70)$ reported that their most significant event was blast only. Because greater than mild injuries were significantly more likely to involve blunt force $\left(\chi^{2}=\right.$ 6.94, $p=.008$ ), we conducted 2 (severity: mild vs. greater than mild) x 2 (mechanism: blunt vs. blast only) ANOVAs with the three DASS-21 scores as the dependent 


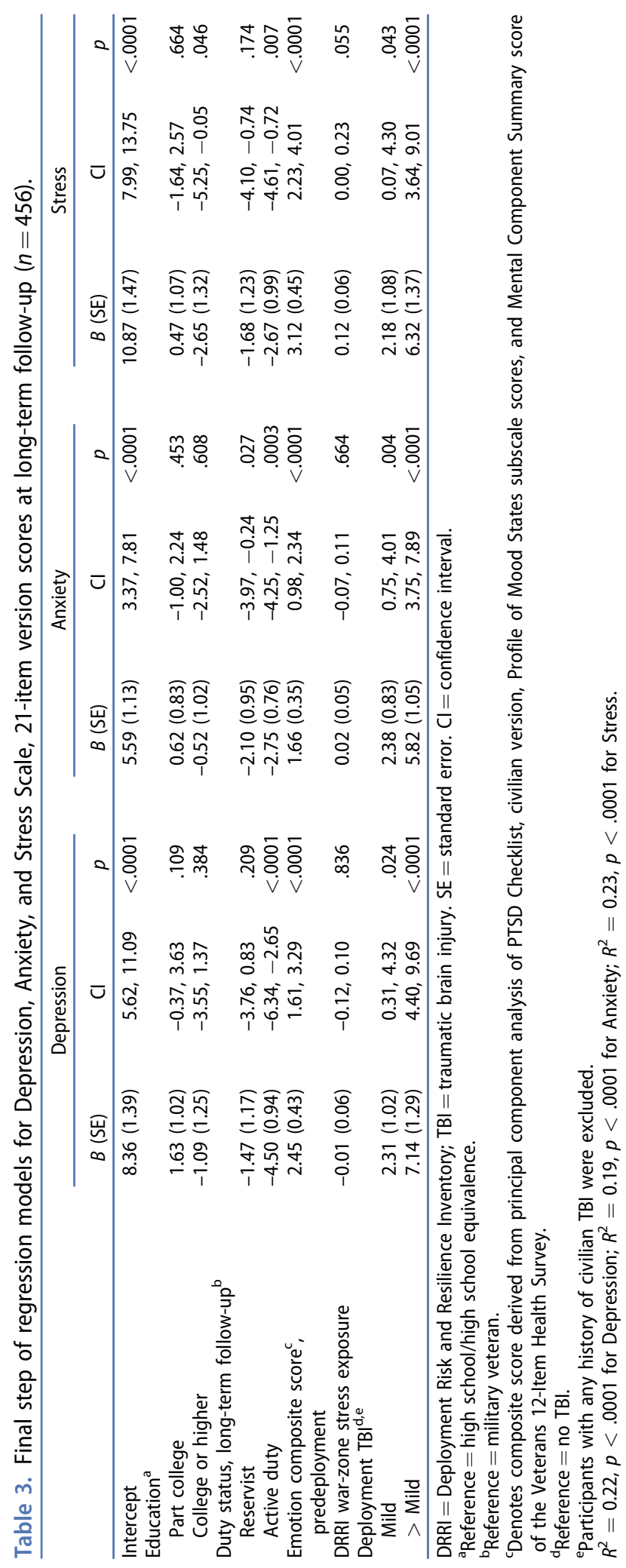


variables, using the Bonferroni-corrected significance level of $p=.017$. Results indicated significant main effects for severity for depression $[F(3,167)=13.15, p=.003]$, anxiety $[F(3,167)=11.18, p=.001]$, and stress $[F(3,167)=13.62, p=.003]$, in which participants reporting TBI events greater than mild in severity endorsed more significant distress at long-term follow-up. None of the main effects for mechanism reached the corrected significance level, although a main effect approached significance for stress $[F(3,167)=4.90, p=.028]$. Similarly, none of the interactions reached the corrected significance level, although the interaction for anxiety approached significance $[F(3,167)=4.43, p=.037]$.

\section{Discussion}

In this prospective study of warzone TBI and long-term emotional distress assessed an average of 8.3 years following return from an index deployment to Iraq, results revealed that $\mathrm{TBI}$ events of greater than mild severity levels were significantly associated with more severe depression, anxiety, and stress symptoms, and those of mild severity were significantly associated with anxiety. Although association of mild TBI with depression and anxiety did not reach our conservative adjustment of $p<.017$, these associations were in the same direction and approached significance $(p>.017$ and $<.05)$. It is noteworthy that results of adjusted analyses indicated that participants who reported their most significant TBI event to be of greater than mild severity endorsed experiencing more significant negative affect at long-term follow-up than those who reported their most significant TBI event to be of mild severity. Our findings, by documenting associations between deployment $\mathrm{TBI}$ and negative component emotional states that constitute these disorders many years following the TBI, complement other military-based studies that have found TBI to be associated with increased risk of mental health disorders (e.g. MacDonald et al., 2014; Stein et al., 2015; Yurgil et al., 2014). Our findings are also consistent with longitudinal studies of civilian TBI that have documented emotional concerns many years after the injury (e.g. Draper et al., 2007).

Because warzone contexts at higher risk for TBI are often stressful, it could be hypothesized that TBI serves as a proxy for warzone stress, and that stress, as opposed to $\mathrm{TBI}$, accounts for greater negative affect among participants who experienced TBI. In our study, and as might be expected, participants reporting TBI also reported more extensive deployment stress exposure, and more extensive stress exposure was associated with higher levels of stress symptoms. TBI, however, was significantly associated with long-term emotional distress even after adjusting for deployment-related stress exposures, suggesting that TBI contributed to risk of long-term emotional distress beyond the stressful contexts in which the TBI was experienced. These findings are consistent with those of Yurgil et al. (2014), who found an association between warzone TBI and subsequent PTSD, after adjusting for combat intensity.

It could also be hypothesized that participants who experienced more emotional distress were more likely to experience or report TBI. In a previous study, we found that higher levels of PTSD symptoms at post-deployment were associated with greater likelihood of inconsistent reports of TBI over time. In the present study, however, we 
excluded participants who reported TBI inconsistently over time. Prior research relatedly suggests that pre-injury psychological concerns are associated with persistent symptom complaints following the injury, including complaints of increased emotional distress (Ponsford et al., 2019). Consistent with this literature, pre-deployment distress in our study was associated with distress at long-term follow-up. Yet, in analyses adjusted for pre-deployment (and, therefore, pre-injury) distress, TBI contributed independently to long-term distress levels, suggesting that results cannot be explained by chronic negative affectivity alone.

Of relevance to public health policy, military veteran status, in addition to TBI, was associated with increased emotional distress at long-term follow-up - a finding consistent with a previous study using an overlapping sample that documented an association between military veteran status and increased risk of PTSD at long-term follow-up (Vasterling et al., 2016). Consistent with the "healthy warrior" construct, it could be hypothesized that service members with less emotional distress are less likely to separate from service than those experiencing more distress. Regardless of the mechanism explaining the association, these findings reinforce the need to provide care for the mental health concerns of military veterans, including both those who experienced TBI and those who did not.

It is also notable that we found associations of greater than mild TBI with three components of emotional distress (i.e. depression, anxiety, and stress) measured by a scale designed to minimize symptom overlap across emotion constructs. These findings suggest that although generalized distress or negative affectivity may be a component of the long-term emotional consequences of TBI, symptoms that differentiate depression from anxiety are elevated in association with greater than mild TBI. In contrast, mild TBI showed a stronger association with anxiety than with depression or stress, although non-significant associations of mild TBI with depression and stress were in the same direction as associations with anxiety. Prior factor-analytic evaluation of the DASS-21 called into question the item composition of the three scales in TBI populations (Wong et al., 2013), but subsequent factor-analytic work has found adequate construct validity for DASS-21 subscales (Randall et al., 2017), as compared with the full DASS. Further, our results are consistent with prior research suggesting that TBI elevates risk of anxiety-, mood-, and stress-related disorders (e.g. O'Donnell et al., 2016; Smith, 2006; Stein et al., 2019; van der Horn et al., 2013; Vasterling et al., 2018).

In exploratory analyses addressing associations of TBI characteristics other than injury severity with emotional outcomes, we failed to detect a significant association with number of TBI events. Prior literature has suggested that repeated TBI leads to cumulative effects that impede recovery (e.g. Guskiewicz et al., 2003), especially after three or more injuries (Iverson et al., 2006), but much of this work has been conducted within the context of sports-related injuries, in which both injuries and non-concussive blows to the head may be numerous and occurring in close temporal proximity. The broader literature is less conclusive (see Yumul \& McKinlay, 2016, for a review). In our study, a minority of participants (33.3\%) reported sustaining three or more injuries. Further, injuries were typically sustained across multiple deployments and therefore potentially allowed for more complete recovery prior to a subsequent injury, thereby less like to invoke physiological mechanisms thought to underlie the 
damaging effects of repeated injuries occurring prior to recovery from previous injury (Giza \& Hovda, 2014). Contrary to studies indicating that blast related TBI may be associated with poorer emotional outcomes than non-blast injuries (e.g. Belanger et al., 2009; Kontos et al., 2013), we did not find a main effect for mechanism of injury in our study. This finding may reflect, however, that due to the small number of participants that reported blunt force only injuries, we compared blast-only injuries to blunt force injuries both with and without associated blast exposures. Therefore, the comparison differed from previous research, which examined blast-related vs. non-blast-related. Moreover, mechanism was partially confounded with severity, with TBI events greater in severity more likely to involve blunt force.

Several limitations of the study should be considered in interpreting the results. First, we sampled only Army soldiers and veterans, therefore limiting generalization of results to other service branches. Women, commissioned officers, and ethnic minorities are underrepresented, also limiting the degree to which results can be generalized to the broader military population. Second, as is common in the Iraq War literature, especially for studies using samples deployed in the earlier stages of the war, TBI history was ascertained on the basis of clinical interview alone. Clinical psychologists, however, conducted the interviews and allowed for interactions to help clarify participant responses, and we excluded participants who reported their index deployment TBI history unreliably over time. Relatedly, although we excluded participants discordant over time in their report of TBI with loss of consciousness, we queried about TBI events with alteration of consciousness only at the long-term follow-up evaluation, and we were therefore not able to assess temporal discordance for events without full loss of consciousness.

Third, we did not administer the DASS-21 pre-deployment, but we were able to construct a pre-deployment emotional distress score from several other measures of emotional wellbeing, including but not limited to the PCL-C as an index of PTSD symptom severity. Fourth, although we employed conservative methods to address two major sources of invalidity (temporal unreliability of TBI report and inconsistent responding within questionnaires), we did not administer a symptom validity test designed to examine possible over- or under-reporting. Finally, previous research with civilians suggests that a broader range of emotional disturbances (e.g. irritability, affective lability) than measured by the DASS-21 may be affected following TBI (Arciniegas et al., 2000) and were therefore not captured in our study.

The prospective design of this study, including pre-deployment assessment of emotional distress, in a sample of sufficient size to examine deployment TBI in participants who had no other history of TBI and measurement of emotional distress at a long-term follow-up, provided a rare opportunity to examine the long-term emotional consequences of deployment TBI. Our findings suggest that TBI increases risk of persistent depression, anxiety, and stress symptoms above and beyond that associated with pre-injury psychological concerns and deployment stress exposures. Clinical implications include appropriate mental health screening of war-zone veterans who experienced deployment $\mathrm{TBI}$, including both those mild and greater than mild in severity. 


\section{Disclosure statement}

The views expressed in this article are those of the authors and do not reflect official policy or position of the US Army, Department of Defense, VA, Food and Drug Administration, or the US government.

There are no conflicts of interest or financial disclosures to report for any of the authors.

\section{Acknowledgments}

We appreciate the support of the Defense Manpower Data Center in obtaining military service status data. We also thank Patricia Crutchfield for her organizational and administrative support.

\section{Funding}

The study described in this manuscript was supported by the Department of Veterans Affairs (VA) Cooperative Studies Program \#566. Support for previous collection of archived data used in this study's longitudinal analyses was provided by the US Army Medical Research and Materiel Command (DAMD 17-03-0020) and VA Clinical Sciences Research and Development.

\section{References}

Alosco, M. L., Aslan, M., Du, M., Ko, J., Proctor, S. P., Concato, J., \& Vasterling, J. J. (2016). Consistency of recall for deployment-related traumatic brain injury. Journal of Head Trauma Rehabilitation, 31(5), 360-368. doi:10.1097/HTR.0000000000000201

Arciniegas, D. B., Topkoff, J., \& Silver, J. M. (2000). Neuropsychiatric aspects of traumatic brain injury. Current Treatment Options in Neurology, 2(2), 169-186.

Aslan, M., Concato, J., Peduzzi, P. N., Proctor, S. P., Schnurr, P. P., Marx, B. P., McFall, M., Gleason, T., Huang, G. D., \& Vasterling, J. J. (2013). Design of neuropsychological and mental health outcomes of operation Iraqi freedom: A longitudinal cohort study. Journal of Investigative Medicine, 61(3), 569-577.

Belanger, H. G., Kretzmer, T., Yoash-Gantz, R., Pickett, T., \& Tupler, L. A. (2009). Cognitive sequelae of blast-related versus other mechanisms of brain trauma. Journal of the International Neuropsychological Society, 15(1), 1-8. doi:10.1017/S1355617708090036

Bryant, R. A., O'Donnell, M. L., Creamer, M., McFarlane, A. C., Clark, C. R., \& Silove, D. (2010). The psychiatric sequelae of traumatic injury. American Journal of Psychiatry, 167(3), 312-320.

Church, H. R., Seewald, P. M., Clark, J. M. R., Jak, A. J., \& Twamley, E. W. (2019). Predictors of work outcomes following supported employment in veterans with a history of traumatic brain injury. NeuroRehabilitation, 44(3), 333-339. doi:10.3233/NRE-182631

Ciarleglio, M. M., Aslan, M., Proctor, S. P., Concato, J., Ko, J., Kaiser, A. P., \& Vasterling, J. J. (2018). Associations of stress exposures and social support with long-term mental health outcomes among US Iraq War Veterans. Behavior Therapy, 49(5), 653-667.

Clark, L. A., \& Watson, D. (1991). Tripartite model of anxiety and depression: Psychometric evidence and taxonomic implications. Journal of Abnormal Psychology, 100(3), 316-336.

Defense and Veterans Brain Injury Center. (2019, July). DoD worldwide numbers for TBI. https:// dvbic.dcoe.mil/dod-worldwide-numbers-tbi

Draper, K., Ponsford, J., \& Schönberger, M. (2007). Psychosocial and emotional outcomes 10 years following traumatic brain injury. Journal of Head Trauma Rehabilitation, 22(5), 278-287. doi:10.1097/01.HTR.0000290972.63753.a7

Giza, C. C., \& Hovda, D. A. (2014). The new neurometabolic cascade of concussion. Neurosurgery, 75(Suppl 4), S24-S33. doi:10.1227/NEU.0000000000000505 
Guskiewicz, K. M., McCrea, M., Marshall, S. W., Cantu, R. C., Randolph, C., Barr, W., Onate, J. A., \& Kelly, J. P. (2003). Cumulative effects associated with recurrent concussion in collegiate football players: The NCAA Concussion Study. JAMA, 290(19), 2549-2555. doi:10.1001/jama.290.19. 2549

Henry, J. D., \& Crawford, J. R. (2005). The short-form version of the Depression Anxiety Stress Scales (DASS-21): Construct validity and normative data in a large non-clinical sample. British Journal of Clinical Psychology, 44(2), 227-239. doi:10.1348/014466505X29657

Iverson, G. L., Brooks, B. L., Lovell, M. R., \& Collins, M. W. (2006). No cumulative effects for one or two previous concussions. British Journal of Sports Medicine, 40(1), 72. doi:10.1136/bjsm.2005. 020651

Kazis, L. E., Wilson, N., Skinner, K., Lee, A., Rogers, W., Ren, X. S., \& Miller, D. (1999). Health status and outcomes of veterans: Physical and Mental Component Summary Scores Veterans SF-12 1998 National Survey of Hospitalized Patients. Center for Health Quality, Outcomes, and Economic Research.

King, L. A., King, D. W., Vogt, D. S., Knight, J., \& Samper, R. E. (2006). Deployment risk and resilience inventory: A collection of measures for studying deployment-related experiences of military personnel and veterans. Military Psychology, 18(2), 89-120.

Kontos, A. P., Kotwal, R. S., Elbin, R. J., Lutz, R. H., Forsten, R. D., Benson, P. J., \& Guskiewicz, K. M. (2013). Residual effects of combat-related mild traumatic brain injury. Journal of Neurotrauma, 30(8), 680-686. doi:10.1089/neu.2012.2506

Lingsma, H. F., Yue, J. K., Maas, A. I. R., Steyerberg, E. W., Manley, G. T., Cooper, S. R., DamsO'Connor, K., Gordon, W. A., Menon, D. K., Mukherjee, P., Okonkwo, D. O., Puccio, A. M., Schnyer, D. M., Valadka, A. B., Vassar, M. J., \& Yuh, E. L. (2015). Outcome prediction after mild and complicated mild traumatic brain injury: External validation of existing models and identification of new predictors using the TRACK-TBI pilot study. Journal of Neurotrauma, 32(2), 83-94.

Lovibond, P. F., \& Lovibond, S. H. (1995). The structure of negative emotional states: Comparison of the Depression Anxiety Stress Scales (DASS) with the Beck Depression and Anxiety Inventories. Behaviour Research and Therapy, 33(3), 335-343.

Ma, H.-P., Chen, P.-S., Wong, C.-S., Chang, C.-F., Ou, J.-C., Tsai, Y.-R., Chiu, W.-T., Tsai, S.-H., Liao, K.-H., Chiang, Y.-H., Wang, J.-Y., Chen, K.-Y., \& Wu, J. C.-C. (2019). Psychometric evaluation of anxiety, depression, and sleep quality after a mild traumatic brain injury: A longitudinal study. Behavioural Neurology, 2019, 1-9. doi:10.1155/2019/4364592

MacDonald, C. L., Johnson, A. M., Nelson, E. C., Werner, N. J., Fang, R., Flaherty, S. F., \& Brody, D. L. (2014). Functional status after blast-plus-impact complex concussive traumatic brain injury in evacuated United States military personnel. Journal of Neurotrauma, 31(10), 889-898.

McNair, D. M., Lorr, M., \& Droppleman, L. F. (1971). Profile of mood states. Educational and Industrial Testing Service.

Newsome, M. R., Scheibel, R. S., Mayer, A. R., Chu, Z. D., Wilde, E. A., Hanten, G., Steinberg, J. L., Lin, X., Li, X., Merkley, T. L., Hunter, J. V., Vasquez, A. C., Cook, L., Lu, H., Vinton, K., \& Levin, H. S. (2013). How functional connectivity between emotion regulation structures can be disrupted: Preliminary evidence from adolescents with moderate to severe traumatic brain injury. Journal of the International Neuropsychological Society, 19(8), 911-924. doi:10.1017/ S1355617713000817

O'Donnell, M. L., Alkemade, N., Creamer, M. C., McFarlane, A. C., Silove, D., Bryant, R. A., \& Forbes, D. (2016). The long-term psychiatric sequelae of severe injury: A 6-year follow-up study. The Journal of Clinical Psychiatry, 77(04), e473-e479. doi:10.4088/JCP.14m09721

Ponsford, J., Cameron, P., Fitzgerald, M., Grant, M., Mikocka-Walus, A., \& Schönberger, M. (2012). Predictors of postconcussive symptoms 3 months after mild traumatic brain injury. Neuropsychology, 26(3), 304-313.

Ponsford, J., Nguyen, S., Downing, M., Bosch, M., McKenzie, J., Turner, S., Chau, M., Mortimer, D., Gruen, R., Knott, J., \& Green, S. (2019). Factors associated with persistent post-concussion symptoms following mild traumatic brain injury in adults. Journal of Rehabilitation Medicine, 51(1), 32-39. 
Randall, D., Thomas, M., Whiting, D., \& McGrath, A. (2017). Depression Anxiety Stress Scales (DASS-21): Factor structure in traumatic brain injury rehabilitation. Journal of Head Trauma Rehabilitation, 32(2), 134-144.

Ruggiero, K. J., Del Ben, K., Scotti, J. R., \& Rabalais, A. E. (2003). Psychometric properties of the PTSD Checklist-Civilian version. Journal of Traumatic Stress, 16(5), 495-502.

Schiehser, D. M., Twamley, E. W., Liu, L., Matevosyan, A., Filoteo, J. V., Jak, A. J., ... DelanoWood, L. (2015). The relationship between postconcussive symptoms and quality of life in veterans with mild to moderate traumatic brain injury. Journal of Head Trauma Rehabilitation, 30(4), E21-E28. doi:10.1097/HTR.0000000000000065

Selim, A. J., Rogers, W., Fleishman, J. A., Qian, S. X., Fincke, B. G., Rothendler, J. A., \& Kazis, L. E. (2009). Updated US population standard for the Veterans RAND 12-item Health Survey (VR12). Quality of Life Research, 18(1), 43-52.

Shields, C., Ownsworth, T., O'Donovan, A., \& Fleming, J. (2016). A transdiagnostic investigation of emotional distress after traumatic brain injury. Neuropsychological Rehabilitation, 26(3), 410-445. doi:10.1080/09602011.2015.1037772

Smith, D. H. (2006). Mild traumatic brain injury and psychiatric illness. British Columbia Medical Journal, 48(10), 510-514.

Spikman, J. M., Timmerman, M. E., Milders, M. V., Veenstra, W. S., \& van der Naalt, J. (2012). Social cognition impairments in relation to general cognitive deficits, injury severity, and prefrontal lesions in traumatic brain injury patients. Journal of Neurotrauma, 29(1), 101-111. doi: 10.1089/neu.2011.2084

Stein, M. B., Jain, S., Giacino, J. T., Levin, H., Dikmen, S., Nelson, L. D., Vassar, M. J., Okonkwo, D. O., Diaz-Arrastia, R., Robertson, C. S., Mukherjee, P., McCrea, M., Mac Donald, C. L., Yue, J. K., Yuh, E., Sun, X., Campbell-Sills, L., Temkin, N., Manley, G. T., ... Zafonte, R. (2019). Risk of posttraumatic stress disorder and major depression in civilian patients after mild traumatic brain injury: A TRACK-TBI study. JAMA Psychiatry, 76(3), 249-258.

Stein, M. B., Kessler, R. C., Heeringa, S. G., Jain, S., Campbell-Sills, L., Colpe, L. J., Fullerton, C. S., Nock, M. K., Sampson, N. A., Schoenbaum, M., Sun, X., Thomas, M. L., \& Ursano, R. J. (2015). Prospective longitudinal evaluation of the effect of deployment-acquired traumatic brain injury on posttraumatic stress and related disorders: Results from the Army Study to Assess Risk and Resilience in Service Members (Army STARRS). American Journal of Psychiatry, 172(11), 1101-1111.

Stulemeijer, M., Vos, P. E., Bleijenberg, G., \& van der Werf, S. P. (2007). Cognitive complaints after mild traumatic brain injury: Things are not always what they seem. Journal of Psychosomatic Research, 63(6), 637-645.

Swan, A. A., Amuan, M. E., Morissette, S. B., Finley, E. P., Eapen, B. C., Jaramillo, C. A., \& Pugh, M. J. (2018). Long-term physical and mental health outcomes associated with traumatic brain injury severity in post-9/11 veterans: A retrospective cohort study. Brain Injury, 32(13-14), 1637-1650. doi:10.1080/02699052.2018.1518539

van der Horn, H. J., Spikman, J. M., Jacobs, B., \& van der Naalt, J. (2013). Postconcussive complaints, anxiety, and depression related to vocational outcome in minor to severe traumatic brain injury. Archives of Physical Medicine and Rehabilitation, 94(5), 867-874.

van der Naalt, J., Timmerman, M. E., de Koning, M. E., van der Horn, H. J., Scheenen, M. E., Jacobs, B., Hageman, G., Yilmaz, T., Roks, G., \& Spikman, J. M. (2017). Early predictors of outcome after mild traumatic brain injury (UPFRONT): An observational cohort study. The Lancet Neurology, 16(7), 532-540. doi:10.1016/S1474-4422(17)30117-5

Vasterling, J. J., Aslan, M., Lee, L. O., Proctor, S. P., Ko, J., Jacob, S., \& Concato, J. (2018). Longitudinal associations among posttraumatic stress disorder symptoms, traumatic brain injury, and neurocognitive functioning in Army soldiers deployed to the Iraq war. Journal of the International Neuropsychological Society, 24(4), 311-323. doi:10.1016/10.1017/ S1355617717001059

Vasterling, J. J., Aslan, M., Proctor, S. P., Ko, J., Marx, B. P., Jakupcak, M., Schnurr, P. P., Gleason, T., Huang, G. D., \& Concato, J. (2016). Longitudinal examination of posttraumatic stress 
disorder as a long-term outcome of Iraq war deployment. American Journal of Epidemiology, 184(11), 796-805.

Vasterling, J. J., Proctor, S. P., Amoroso, P., Kane, R., Gackstetter, G., Ryan, M. A., \& Friedman, M. J. (2006). The Neurocognition Deployment Health Study: A prospective cohort study of army soldiers. Military Medicine, 171(3), 253-260.

Vasterling, J. J., Proctor, S. P., Aslan, M., Ko, J., Jakupcak, M., Harte, C. B., Marx, B. P., \& Concato, J. (2015). Military, demographic, and psychosocial predictors of military retention in enlisted army soldiers 12 months after deployment to Iraq. Military Medicine, 180(5), 524-532.

Vogt, D. S., Proctor, S. P., King, D. W., King, L. A., \& Vasterling, J. J. (2008). Validation of scales from the Deployment Risk and Resilience Inventory in a sample of Operation Iraqi Freedom veterans. Assessment, 15(4), 391-403.

Waldron-Perrine, B., Hennrick, H., Spencer, R. J., Pangilinan, P. H., \& Bieliauskas, L. A. (2014). Postconcussive symptom report in polytrauma: Influence of mild traumatic brain injury and psychiatric distress. Military Medicine, 179(8), 856-864.

Wäljas, M., Iverson, G. L., Lange, R. T., Hakulinen, U., Dastidar, P., Huhtala, H., Liimatainen, S., Hartikainen, K., \& Öhman, J. (2015). A prospective biopsychosocial study of the persistent post-concussion symptoms following mild traumatic brain injury. Journal of Neurotrauma, 32(8), 534-547.

Williams, M. W., Rapport, L. J., Millis, S. R., \& Hanks, R. A. (2014). Psychosocial outcomes after traumatic brain injury: Life satisfaction, community integration, and distress. Rehabilitation Psychology, 59(3), 298-305.

Wong, D., Dahm, J., \& Ponsford, J. (2013). Factor structure of the depression anxiety stress scales in individuals with traumatic brain injury. Brain Injury, 27(12), 1377-1382.

Yumul, J. N., \& McKinlay, A. (2016). Do multiple concussions lead to cumulative cognitive deficits? A literature review. PM\&R, 8(11), 1097-1103. doi:10.1016/j.pmrj.2016.05.005

Yurgil, K. A., Barkauskas, D. A., Vasterling, J. J., Nievergelt, C. M., Larson, G. E., Schork, N. J., Litz, B. T., Nash, W. P., \& Baker, D. G. (2014). Association between traumatic brain injury and risk of posttraumatic stress disorder in active-duty Marines. JAMA Psychiatry, 71(2), 149-157. doi:10. 1001/jamapsychiatry.2013.3080 